\title{
NOTE ON THE COORDINATION OF PERIODIC PROCESSES IN TRANSPORTATION SYSTEMS
}

The article deals with a coordination of periodic processes in transport systems. It introduces some basic mathematical models useful in process optimization. It also shows some examples of everyday transport practices where this optimization can be used.

\section{Introduction}

Users of transport systems often declare their interest in velocity. However, it does not mean technical velocity of transport means. They concern the "velocity of displacement", i.e. the distance between the origin and destination divided by the transport time. And it is often true that this value is strongly influenced by coordination between transportation processes.

Let us consider several examples:

A. A passenger travels from the village $V$ to the town $W$ using first a local train from $V$ to a station $S$ and afterwards an express train from $S$ to $W$. It is obvious that the travel time from $V$ to $W$ is strongly influenced by the waiting time for the train changing in $S$ and, consequently, by the coordination of the transport processes of local and express train operation.

B. A passenger walks from his house $H$ to an urban bus stop $S_{1}$, then he/she travels by bus from the stop $S_{1}$ to the stop $S_{2}$, using any from the two routes $r_{1}, r_{2}$ operating between $S_{1}$ and $S_{2}$. It is obvious that the total duration of the trip from $H$ to $S_{2}$ is strongly influenced by the waiting time for a bus at the stop $S_{1}$ and, consequently, by the coordination of the transport processes on the two routes.

C. A wagon is loaded at a station $A$ and its destination is the station $B$. However, it has first to use a train to a marshalling yard $Y$ and after some manipulations there to use another train from $Y$ to $B$. It is obvious that the total duration of the trip from $A$ to $B$ is strongly influenced by the manipulation time in $Y$ and, consequently, by the coordination of the processes in $Y$.

D. A person drives his car from his home $H$ to his office $O$ passing through a signalized road intersection $I$. It is obvious that the total duration of the trip from $H$ to $O$ is strongly influenced by the waiting time in front of the junction $I$ and, consequently, by the coordination of the processes at I.

If we consider the cases A., B. and D. we can imagine that the displacement activities may be more complicated:

A. There may exist many local trains coordinated with several express trains in several stations.
B. There may exist many routes coordinated at many common legs.

D. There may exist many processes at several junctions to be coordinated.

The complex problems of optimal coordination can be a bit simplified introducing a periodicity:

A. To introduce a periodic timetable (a "Takt Fahrplan" in German), e.g. to repeat the departures after each hour.

B. To introduce a periodic time table, e.g. to repeat the departures after each 12 minutes.

C. To introduce a periodic schedule, e.g. to repeat the processes each day (it was already done in the major part of marshalling yards).

D. To introduce a "cyclic" mode of operation, i.e. to repeat the green signals e.g. each 80 seconds (a big part of junctions work in a periodic regime now).

In the sequel we shall try to formulate the coordination problems in a general mathematical way.

\section{Basic mathematical models}

One can find many different periodic point processes (more precisely: time-point processes) in transportation systems, e.g.:

A. The departures of express trains from the station $S_{1}$ for the station $S_{2}$ are scheduled for $6: 42,7: 42,8: 42$ etc. This defines a point process $p_{2}=6: 42,7: 42,8: 42, \ldots=6: 42+k \times 1: 00$, $k=1,2, \ldots$ (the reason for using $\mathrm{p} 2$ will be seen later)

B. The departures of urban transport buses from the stop $S_{1}$ for the stop $S_{2}$ are scheduled for 6:06, 6:18, 6:30, 6:42, etc. $p_{1}=$ $=6: 06,6: 18,6: 30,6: 42, \ldots=.6: 06+k \times 0: 12, k=1,2, \ldots$

C. Wagon collection process on the sorting siding $s_{1}$ of a marshalling yard is concluded each 6 hours at 3:20, 9:20, 15:20, $21: 20 . p_{1}=3: 20,9: 20, \ldots=3: 20+k \times 6: 00, k=1,2, \ldots$

D. At a signalized road intersection $I$, the green light start each 80 seconds for the given stream $S_{1}$ of vehicles e.g. $p_{1}=$ $=5: 00: 12+k \times 0: 1: 20, k=1,2, \ldots$

\footnotetext{
* Jan Černý, Vlastislav Mojžíš

Jan Perner Transport Faculty, University Pardubice, Studentská 95, CZ-532 10 Pardubice, Czech Republic, E-mail: vlastislav.mojzis@upce.cz
} 
Usually, the periodic point processes are not isolated. On the contrary, there exist sets of mutually influenced processes and, usually again, the processes have either the same period, or the least common multiple of the periods, which is not very much greater than they are. For instance:

A. Besides the process $\mathrm{p} 2$ we have another process $p_{1}=6: 28$, $6: 58,7: 28, \ldots=6: 28+k \times 0: 30$ representing the arrivals of local trains to the station $S_{1}$ from the station $S_{3}$. In this case, the corresponding periods are $p_{2}=1: 00=60$ min., $p_{1}=0: 30$ $=30 \mathrm{~min}$., the common multiple $p=60 \mathrm{~min} .=2 \times p_{1}=$ $=1 \times p_{2}$.

B. Besides the process $p_{1}$, corresponding to a route $r_{1}$ we have another process $p_{2}=6: 00,6: 20,6: 40, \ldots=6: 00+k \times 0: 20$ representing the departures of buses of another route $\mathrm{r} 2$ from the stop $S_{1}$ for the stop $S_{3}$ via the stop $S_{2}$. In this case, the corresponding periods are $p_{1}=0: 12=12 \mathrm{~min} ., p_{2}=0: 20=20$ min., the common multiple $p=60 \mathrm{~min} .=5 \times p 1=3 \times p 2$.

C. Besides the process $p_{1}$, corresponding to the sorting siding $s_{1}$ we have another process $p_{2}=1: 30,9: 30, \ldots=1: 30+k \times 8: 00$ corresponding to the sorting siding $s_{2}$. In this case, the corresponding periods are $p_{1}=6: 00=6 h ., p_{2}=8: 00=8 h$., the common multiple $p=24$ min. $=4 \times p_{1}=3 \times p_{2}$.

D. Besides the process $p_{1}$, corresponding to the stream $S_{1}$ we have another process $p_{2}=5: 00: 45+k \times 0: 1: 20, k=1,2, \ldots$ corresponding to another stream $S_{2}$, which is in collision with $S_{1}$. Here $p_{1}=p_{2}=p=80$ seconds.

\section{Coordination}

Having two processes at the same place it is quite natural to require some type of coordination between them. In general, we can meet different requirements:

2.1. Single and simple linking. We speak about single linking if we have two processes $p_{1}, p_{2}$ with only one linking between them, e.g. $p_{2}$ linked to $p_{1}$, symbolically $p_{1} \rightarrow p_{2}$. We call this linking simple if both processes have the same number $\mathrm{n}$ of time-points in one period $\mathrm{p}$ and the process $p_{2}=t_{21}, t_{22}, \ldots, t_{2 n}(+k p)$ is linked to the process $p_{1}=t_{11}, t_{12}, \ldots, t_{1 n}(+k p)$. The quality of this linking can be expressed by the differences $d_{1}=t_{21}-t_{11}, d_{2}=$ $=t_{22}-t_{12}, \ldots, d_{n}=t_{2 n}-t_{1 n}$ put into an objective function $f\left(d_{1}\right.$, $\left.d_{2}, \ldots, d_{n}\right)$, e.g.

$$
\begin{aligned}
& f_{1}\left(d_{1}, d_{2}, \ldots, d_{n}\right)=\min \left\{d_{1}, d_{2}, \ldots, d_{n}\right\} \text { (greater is better) } \\
& f_{2}\left(d_{1}, d_{2}, \ldots, d_{n}\right)=\max \left[d_{1}, d_{2}, \ldots, d_{n}\right\} \text { (smaller is better) } \\
& f_{3}\left(d_{1}, d_{2}, \ldots, d_{n}\right)=\max \left[d_{1}, d_{2}, \ldots, d_{n}\right]-\min \left\{d_{1}, d_{2}, \ldots, d_{n}\right\} \\
& (\text { smaller is better) } \\
& f_{4}\left(d_{1}, d_{2}, \ldots, d_{n}\right)=d_{1}^{2}+d_{2}^{2}+\ldots+d_{n}^{2} \text { (smaller is better). }
\end{aligned}
$$

Among the abovementioned examples only the A can serve as the illustration of single linking, but, unfortunately, it is not simple (we have 2 arrivals within $60 \mathrm{~min}$. but only one departure).

2.2. Double and multiple linking. We speak about double linking if:

a) We have both $p_{1} \rightarrow p_{2}$ and $p_{2} \rightarrow p_{1}$. b) There exists another pair $p_{3} \rightarrow p_{4}$.

In the case a) we speak about double mutual linking of the pair $p_{1}, p_{2}$

We speak about multiple linking if there exist more than one linked pair (i.e. double linking is a particular case of multiple linking). We suppose we are given a set of time-point periodic processes $P=\left\{p_{1}, p_{2}, \ldots, p_{m}\right\}$ with periods $p_{1}, p_{2}, \ldots, p_{m}$ and the common period $p=\mu\left(p_{1}, p_{2}, \ldots, p_{m}\right)=$ the least common multiple of the periods $p_{1}, p_{2}, \ldots, p_{m}$. The mutual linking could be expressed by means of a linking digraph (= oriented graph) $G=(V, A)$, where the vertex set $V=\{1,2, \ldots, m\}$ represents the processes $p_{1}$, $p_{2}, \ldots, p_{m}$, the $\operatorname{arc} \operatorname{set} A$ represents linking, i.e. the $\operatorname{arc} a=(i, j) \in A$ represents linking $p_{j}$ to $p_{i}$ i.e. $p_{i} \rightarrow p_{j}$.

Writing the time-point one has to take into account the common period $p$, i.e. if a time-point is represented by a number $t_{i k}$ then $t_{i k} \in\{0,1, \ldots, \mathrm{p}\}$ must hold; if not, then it must be reduced mod $p$

2.3. General single linking. We speak about general single linking $p_{1} \rightarrow p_{2}$ if it is not simple. In the case of general single linking the processes $p_{1}, p_{2}$ may have different numbers $n_{1}, n_{2}$ of time-points in one period $p$ and the differences $d_{i}$ may be calculated from some selected $n$-tuples $t_{11}, t_{12}, \ldots, t_{1 n}, t_{21}, t_{22}, \ldots, t_{2 n}, n \leq n_{1}$, $n \leq n_{2}$.

Especially, if the linking $p_{1} \rightarrow p_{2}$ intends to express a train changing, we denote $T_{1}$ the set of all time-points (= arrivals to the change station plus some time for walking from one train to another) of the process $p_{1}$ in one period $p$ and $T_{2}$ is the set of all time-points (departures from the change station) $p_{2}$, but moreover both the sets $T_{1}, T_{2}$ are extended by adding the first time-point from the next period (i.e. we add the time-point $t+p$ where $t$ is the first time-point from the period $p$ ). Then the number $n$ is the maximum natural number allowing the $n$-tuples $t_{11}, t_{12}, \ldots, t_{1 n}, t_{21}$, $t_{22}, \ldots, t_{2 n}$ to have the following properties of "closeness": $t_{1 i}=$ $=\max \left[t \in T_{1}: t \leq t_{2 i}\right], t_{2 i}=\min \left[t \in T_{2}: t \geq t_{1 i}\right]$. If in such a manner $t_{1 n}=t_{11}+p$ and $t_{2 n}=t_{21}+p$ then we omit $t_{1 n}, t_{2 n}$ and we put $n-1$ instead of $n$.

In our example $A$ we have $T_{1}=\{28,58,88\}, T_{2}=\{42,102\}$ corresponding to the local train arrivals $6: 28,6: 58,7: 28$ and the express train departures 6:42, 7:42. Obviously, the first value of $n=2, t_{11}, t_{12}=28,88, t_{21}, t_{22}=42,102$. However, $p=60$ and both $88=28+p, 102=42+p$. Thus, we omit 88,102 and put $n=1 . t_{11}=28, t_{22}=42, d_{1}=14$.

2.4. General multiple linking. We suppose we are given a set of time-point periodic processes $P=\left\{p_{1}, p_{2}, \ldots, p_{m}\right\}$ with periods $p_{1}, p_{2}, \ldots, p_{m}$ and the common period $p=\mu\left(p_{1}, p_{2}, \ldots, p_{m}\right)$ and the mutual linking expressed by means of a linking digraph $G=(V$, $A$ ). In the case of general multiple linking we suppose there exists a general rule determining the number $\mathrm{n}$, the values $d_{1}, d_{2}, \ldots, d_{n}$ and the objective function $f$ expressing the quality of linking by means of the value $f\left(d_{1}, d_{2}, \ldots, d_{n}\right)$. 
In practice it can happen that these processes are originated in different locations and their influence represented by a relation $p_{i} \rightarrow p_{j}$ "works" in a third location which needs some equalization of time-points. Usually, we use a value $o(i, j)$ said the offset which has to be added to the time-points of the process $p_{i}$ before their comparison with the time-points of $p_{j}$.

The practical meaning of the offset can be demonstrated on our examples:

A. Let us suppose that the departures of local trains from the station $S_{3}$ are 5:38, 6:08, 6:58, $\ldots=5: 38+k \times 0: 30$ and let the running time from $S_{3}$ to $S_{1}$ be $r\left(S_{3}, S_{1}\right)=50 \mathrm{~min}$. Then the arrivals to $S_{1}$ are $6: 28,6: 58,7: 28, \ldots=6: 28+k \times 0: 30$ as we supposed above. Moreover, let the departures of express trains from their origin station $S_{0}$ be 5:32, 6:32, 7:32 etc., i.e. $5: 32+k \times 1: 00$, just $r^{\prime}\left(S_{0}, S_{1}\right)=1: 10 \mathrm{~h} .=70 \mathrm{~min}$. before their departures from $S_{1}$. Then we can consider the original processes $p_{1}=5: 38+k \times 0: 30, p_{2}=5: 32+k \times 1: 00$, the offset $o(1,2)=r\left(S_{3}, S_{1}\right)-r^{\prime}\left(S_{0}, S_{1}\right)+w_{12}=50-70+5=$ $=-15 \mathrm{~min}$. Hence the process $p_{2}=5: 32+k \times 1: 00=$ $=32+60 k$ (with one departure during the common period $p=60$ min.) will be linked to the reduced process $p_{r 1}=5: 38+$ $+k \times 0: 30+o(1,2)=38+30 k-15=23+30 k$ (with two departures during the common period $60 \mathrm{~min}$.). Further calculations will be similar to the ones at the end of the part 2.3.

B. Similarly, the original processes can be represented by the departures from the terminals of the routes, but the offset will be exactly the difference of running times from the terminals to $S_{1}$ without any further correction. Let, for the simplicity, $S_{1}$ be the terminal for both routes. The "general rule" is the following: Let $T_{1}=\left\{t_{1}, t_{2}, \ldots, t_{n}\right\}$ be the set of all departures of all routes from $S_{1}$ to $S_{2}$ within one period $p$. Suppose that $t_{1} \leq \ldots, \leq t_{n}$. Let $t_{n+1}=t_{1}+p$. Then $d_{i}=t_{i+1}-t_{i}, i=1, \ldots$, $n$. The values $d_{1}, d_{2}, \ldots, d_{n}$ represent the waiting intervals of the passengers using the segment $S_{1}, S_{2}$ only.

In our example $d_{1}, \ldots, d_{8}=6,12,2,10,10,2,12,6$ and:

- $f_{1}(6,12,2,10,10,2,12,6)=\min \{6,12,2,10,10,2,12$, $6]=2$ expresses the danger of collision of two subsequent vehicles at the same stop,

- $f_{2}(6,12,2,10,10,2,12,6)=\max [6,12,2,10,10,2,12$, $6\}=12$ expresses the maximum waiting time of a passenger, - $0,5 q f_{4}(6,12,2,10,10,2,12,6)=0,5 q(36+144+4+$ $+100+100+4+144+36)=284 q$ expresses for one period the total waiting time (in minutes) of the q passengers, boarding the vehicles during one minute.

C. On the contrary, here no running time will have to be considered. Instead, the offset $o(i, j)$ will represent the transfer time for employees and engines to move from the $i$-th siding to the $j$-th one after having finished the works transforming wagons into a train. The general rule for the calculation of the values di is similar to the previous one. In our example, neglecting offsets, we have (in minutes) $d_{1}, \ldots, d_{7}=110,360,10,350$, $130,230,250$, but the only objective function having a practical sense is:

- $f_{1}\left(d_{1}, \ldots, d_{7}\right)=\min [110,360,10,350,130,230,250\}=10$ It expresses the shortage of time necessary for the train creation from the wagons on the siding $s_{1}$ before the start of the same works at the siding $s_{2}$.

D. If the processes $p_{i}$ and $p_{j}$ work at the same intersection then the offset $o(i, j)$ will represent the duration of green signal for the stream Si plus the clearing time. If they work at different intersections then the running time between them will have to be added.

\section{Coordination for changes}

Let us turn to our examples. B) concerns the well known problem of coordination of public transport on common legs. C) deals with freight train formations and the methods of solution are similar to the previous ones. D) is the well known problem of signalised intersections. All three are described by many authors in many books and papers, see. e.g. the monograph [1]. One can say that the available methods satisfy the practical needs.

On the other hand the problem A) cannot be considered satisfactorily solved. In [1] and [2] one can find a heuristics and a linear programming model for time shift optimization, having given the set of trains operating on a general network. Other authors, e.g. [3], study the same problem, but limited to the "herring-bone type of network" using congruency calculations.

However, the authors have not yet met any paper dealing with the coordination problems, optimizing:

a) the size of trains for the given number of trains during the common period together with their time shifts

b) the number of trains during the common period together with their size and time positions.

The authors hope they have an idea of solution of the problem a) using mathematical programming. The problem $b$ ) seems to be open.

\section{Acknowledgments}

The research work was partially supported by the grant GAČR No. 103/05/2043

\section{References}

[1] ČERNÁ, A., ČERNÝ, J.: Theory of management and decision making in transportation systems (monograph in Czech). Jan Perner Institute, Prague, 2004.

[2] ČERNÁ, A.: Coordination of public transport timetables in change nodes (in Czech). Res. R. II FES UPa (2003), p. 44-47.

[3] SALZBORN, F. J. N.: Scheduling bus systems with interchanges. Transp. Sci. 14 (1980), p. 211-231.

[4] MOJŽÍŠ, V.: Quality of Transport Processes (in Czech). Jan Perner Institute, Pardubice, 2003. ISBN 80-86530-09-4. 